\title{
Retinal Vascular Tortuosity in Hospitalized Patients with Type 2 Diabetes and Diabetic Retinopathy in China
}

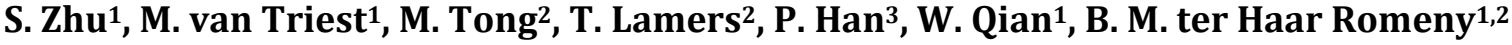 \\ ${ }^{1}$ Biomedical and Information Engineering, Northeastern University, Shenyang, China \\ ${ }^{2}$ Biomedical Engineering, Eindhoven University of Technology, Eindhoven, The Netherlands \\ ${ }^{3}$ Shengjing Hospital of China Medical University, Shenyang, China \\ Email: zhu.shanshan111@outlook.com,miekevantriest@hotmail.com,tong.meng@outlook.com, T.Lamers@student.tue.nl, \\ hanping85@hotmail.com,wqian@bmie.neu.edu.cn, bart.romeny@outlook.com
}

How to cite this paper: Zhu, S., van Triest, M., Tong, M., Lamers, T., Han, P., Qian, W. and ter Haar Romeny, B.M. (2016) Retinal Vascular Tortuosity in Hospitalized Patients with Type 2 Diabetes and Diabetic Retinopathy in China. J. Biomedical Science and Engineering, 9, 143-154. http://dx.doi.org/10.4236/jbise.2016.910B019

Received: August 30, 2016 Accepted: September 20, 2016 Published: September 23, 2016

\begin{abstract}
Context: Diabetic patients are at high risk for microvascular complications of disease such as diabetic retinopathy (DR) and diabetic neuropathy. Imaging the retinal microvasculature offers a chance to measure quantitatively the microvascular changes in diabetic patients' onset and progression. However, the relation between retinal biomarkers and diabetic risk factors is unclear in Chinese hospitalized type 2 diabetic patients. Aims: To examine the associations of retinal vascular tortuosity with risk factors of type 2 diabetes and diabetic retinopathy in Chinese hospitalized patients. Patients and Methods: Our cross-sectional study includes 504 participants with type 2 diabetes hospitalized in the department of endocrinology in Shengjing hospital, Shenyang, China. Patients' socio-demographic, clinical and biological information was retrieved from their ID card, the interview, and the local hospital information system. Retinal photographs were taken by laser-scanning ophthalmoscopy of both eyes and checked if gradable for analysis. The weighted mean and standard deviation of tortuosity were calculated from the retinal photographs using a novel robust and fully automatic quantitative method. DR was assessed from the retinal photographs by the ophthalmologist according to the modified Airlie House classification into No DR and Any DR in our current study. Data were analyzed by SPSS Version 22 with Student's $t$ test, Mann-Whitney $U$ test, Chi-square test, and linear regression. Results: For the total participants (504) in this study, the weighted mean and standard deviation (SD) of tortuosity was $12.05 \times 10^{-3}$ (SD: $\left.1.66 \times 10^{-3}\right)$ and $24.31 \times 10^{-3}$ (SD: $3.69 \times$ $\left.10^{-3}\right)$, respectively. $386(76.6 \%)$ patients were diagnosed with DR who had older age, long duration of diabetes, higher brachial ankle pulse wave velocity (baPWV), higher systolic blood pressure (SBP), higher diastolic blood pressure (DBP), and higher re-
\end{abstract}


tinal vascular tortuosity values. In univariable linear regression analyses, older age, longer duration, higher baPWV, higher urine microalbuminuria, higher urine albu$\mathrm{min} /$ creatinine ratio, diagnosed with high blood pressure, thrombosis or Any DR, were significantly associated with both higher tortuosity measures (all $P$ values < $0.05)$. In multivariable-adjusted linear regression analyses, age and diabetes duration (all $P$ values $<0.05$ ) were independently and positively associated with both tortuosity measures, after adjustment for low-density lipoprotein (LDL), high-density lipoprotein (HDL) and high blood pressure. Furthermore, after adding some kidney failure factors, the urine albumin/creatinine ratio was also an independent risk factor of vascular tortuosity. These associations remained even after including or excluding the factor of DR. Conclusions: Retinal vascular tortuosity is independently associated with older age, longer duration, and higher urine albumin/creatinine ratio in Chinese hospitalized type 2 diabetic patients, regardless of the presence of DR. This suggests that retinal vascular tortuosity might be a successful early indicator of diabetic microvascular complications, like diabetic retinopathy and diabetic neuropathy, and that the precise quantitative measurement of retinal vascular tortuosity is an effective biomarker in the screening and assessment of diabetic microvascular complications.

\section{Keywords}

Retinal Vascular Tortuosity, Diabetic Retinopathy, Diabetic Neuropathy, Type 2 Diabetes, Risk Factor, Biomarker

\section{Introduction}

\subsection{Background}

The epidemic of diabetes is in an explosive increase worldwide, especially in developing countries. In China, according to the study of $\mathrm{Hu}$ [1], the economic development and urbanization brought broad changes in lifestyle and led the fast rising trend of diabetes. A recent study of $\mathrm{Xu}$ et al. (2013) reported the prevalence of diabetes is an alarming $11.6 \%$ in Chinese adults, and 50.1\% have prediabetes [2].

Diabetic patients are at high risk for microvascular complications of the disease such as diabetic retinopathy (DR) and diabetic neuropathy (DN) [3]. Diabetic retinopathy is found to be a leading cause of preventable blindness in working-aged people. In addition, diabetic nephropathy (kidney disease) is on the rise in China. Moreover, type 2 diabetic patients have a relatively short period before developing diabetic nephropathy compared to type 1 diabetes with 10 - 15 years. Although the specific mechanisms underlying the pathology of these microvascular complications remain uncertain, screening for microvascular changes is essential. An easy and cost-effective way is digital fundus photography. The retinal microvasculature is the only part of the human circulation system available for direct and non-invasive optical observations, and offers an opportunity to measure quantitatively the microvascular changes in diabetic patients [4], with a resolution far higher than MRI or CT. 
Further recognition and exploitation of diabetes-associated changes in retinal microvasculature is promising. Many studies have demonstrated the increasing interest in one of the geometric features of the retinal microvasculature, retinal vascular tortuosity (curvature), which might not only be sensitive to early hemodynamic changes in diabetes, but also is more easily observable than other retinal vascular parameters [5]-[7].

\subsection{Previous Research}

Prior studies showed that the relation between tortuosity and having diabetes is less consistent. One study conducted by Sasongko et al. found the retinal vascular tortuosity is positively associated with having diabetes [5], while Cheung et al. showed a negative relationship [8]. This contradiction may be from the population difference including race, age, duration of diabetes and HbAlc.

A few studies have shown some associations between tortuosity and diabetes risk factors. Cheung et al. investigated retinal vascular tortuosity is positively associated with age, BMI and blood pressure [9], Sasongko et al. found that retinal vascular tortuosity is associated with high $\mathrm{HbAlc}$, even before the presence of retinopathy [10]. It suggests that changes in retinal vascular tortuosity may be an early pathology indicator before the clinical manifestation of diabetic microvascular complications. There has also been evidence showing that tortuosity is associated with diabetes microvascular complications, like diabetic retinopathy (DR) and kidney dysfunction [11]-[13].

However, these associations are not investigated in Chinese hospitalized type 2 diabetic patients yet. In addition, the quantitative measuring method of retinal vascular tortuosity, also for much smaller vessels, in our study was different from the way it was measured in prior studies [9] [10].

\subsection{Objective}

In this study, we aimed to investigate the associations between retinal vascular tortuosity, using a novel, robust, fully automatic and highly precise quantitative method in retinal fundus photographs taken by a laser-scanning ophthalmoscope, and a range of risk factors for diabetes and diabetic retinopathy, in a typical sample of Chinese hospitalized patients with type 2 diabetes.

\section{Materials and Methods}

\subsection{Study Participants}

The Shengjing Study is set up in November 2014, which is a cross-sectional survey of diabetic patients who were hospitalized in the department of endocrinology in Shengjing hospital in Shenyang, China. In this report, we selected 504 hospitalized type 2 diabetic patients who took the retinal photographs, gradable for analysis, between January 2015 and May 2015. Written informed consent from each patient was obtained.

\subsection{Data Collection}

Patients' demographic information such as name, date of birth, gender were obtained 
from the patients' identity (ID) card by a self-designed reader system. Other socio-demographic factors including patients' occupation, marital status, and the approximate date of diagnosis of diabetes were asked by interview and recorded in the same database as the ID card information. Diabetes duration was considered the period from diagnosis of diabetes by the retinal photographs taken.

The previous history of hypertension, thrombosis, cardiovascular disease, smoking history, drink history were available from the patients' hospitalized admission record of the hospital information system (HIS). Clinical results such as fasting blood glucose level, serum cholesterol, triglycerides, High-Density Lipoprotein (HDL), Low-Density Lipoprotein (LDL), creatinine, HbAlc, and urine microalbumin, acquired during the hospitalization, were also retrieved from the HIS. If one medical examination was performed multiple times, the results from the first test were used in the statistical analysis.

The patients' systolic blood pressure (SBP), diastolic blood pressure (DBP), and brachial ankle pulse wave velocity (baPWV) were measured with a non-invasive vascular screening device (model BP-203RPEIII, Omron Healthcare Europe B.V., Hoofddorp, the Netherlands). Body mass index (BMI) was calculated from weight (kg) divided by square height $\left(\mathrm{m}^{2}\right)$ from the record information before this examination. We took the blood pressure of the right arm for analysis.

Retinal photographs were taken from both eyes by following a standardized protocol, e.g. nonmydriatic and fovea-centered, using an innovative and portable laser-scanning ophthalmoscope (SLO) camera (EasyScan, by i-Optics Inc., The Hague, the Netherlands). We assessed whether the photographs were gradable or not according to the quality criteria including the amount of noise in the photographs, the presence of artifacts, whether the algorithm was able to find the optic disc and whether it found enough vessels to calculate the tortuosity. The photograph of the right eye was used for analysis, but if it was not gradable, the photograph of the left eye was used.

\subsection{Measurement of Retinal Vascular Tortuosity}

Retinal vascular tortuosity is measured by a novel robust and fully automatic quantitative method developed by E. Bekkers et al. [14]. This brain-inspired method is based on the theory of best-fit exponential curves in the roto-translation group SE (2) developed by Duits, Franken and Janssen [15]-[17], and, importantly, does not rely on explicit segmentation of the blood vessels. 2D images are lifted to 3D functions called "orientation scores" by adding an orientation dimension to the domain [18]. In this newly extended domain of positions and orientations (identified with SE (2)), we study the exponential curves whose curvatures are constant. At each location a curvature and confidence value are computed from tangent vectors of exponential curves that locally best fit the data [16]. Then these values are used to define global tortuosity measures from the histogram.

In this paper, two tortuosity features were calculated: the weighted mean (mean of absolute values) and the standard deviation of all the tortuosity values in one image. Tortuosity was taken for arteries and veins together [14]. 


\subsection{Detection of DR}

Retinopathy was assessed from the retinal photographs by the ophthalmologist according to the ETDRS adaptation of the modified Airlie House classification [19]. Retinopathy was defined as at least 1 micro-aneurysm or hemorrhage in either eye. In the current study, only the classification with or without retinopathy of diabetic patients was evaluated.

\subsection{Statistical Analysis}

Descriptive analyses for participants' characteristics were performed, and expressed as mean \pm SD for continuous variables, and number (percentage) for categorical variables. Since not all the characteristics were known for all the patients, the total number of patients is bigger than the number of patients per characteristic.

We used Student's $t$ test for continuous and normally distributed variables, the Mann-Whitney $U$ test for continuous and skewed variables, and the Chi-square test for categorical variables to assess differences in means or proportions between type 2 diabetic patients with and without DR.

In the linear regression analysis, the weighted mean and the standard deviation of the tortuosity were regarded as dependent variables. Both of these variables were checked for normality using the Kolmogorov-Smirnov test. First, univariable regression analysis was performed with a general linear regression model. Furthermore, we constructed three models for the multivariable regression analysis: in model 1, the variables age, duration of diabetes, HBP, HDL, and LDL were added; model 2 additionally included the urine albumin/creatinine ratio, blood creatinine and DR; model 3 included all variables in model 2 after excluding subjects with DR.

All statistical analyses were carried out using IBM SPSS Statistics Version 22. The significant level for the above mentioned statistical analyses was set as $\alpha=0.05$, and a $P$ value of $<0.05$ (two-tailed) was considered as statistically significant.

\section{Results}

\subsection{Baseline Characteristics and Comparison of Parameters between DR Groups}

A total of 504 hospitalized patients with type 2 diabetes attending the department of endocrinology in Shengjing hospital was studied. There were 270 males (57.6\%) and 199 females (42.4\%) with mean age of $51.4 \pm 12.8$ years and diabetic duration of $6.97 \pm$ 6.57 years. $10.3 \%$ of the patients had cerebral vascular disease (CVD); $39.7 \%$ of patients were diagnosed with high blood pressure; $35.3 \%$ of patients were smokers; and $18.3 \%$ were drinkers. Furthermore, the fasting glucose was $9.32 \pm 3.45 \mathrm{mmol} / \mathrm{L}$; and $34.3 \%$ of patients were with urine microalbuminuria $>1.9 \mathrm{mg} / \mathrm{dL} ; 27.8 \%$ of patients had a urine albumin/creatinine ratio $>30$.

Of all the patients, 386 (76.6\%) were diagnosed with DR. Comparisons between the two groups (No DR and Any DR) revealed that patients with DR had older age, longer 
duration of diabetes, higher baPWV, higher SBP, higher DBP, and were more likely to have higher retinal vascular tortuosity values (weighted mean, standard deviation). In contrast, no significant difference was found between the two groups for HbAlc, BMI, cholesterol, fasting glucose, urine albumin/creatinine ratio, triglyceride, LDL, HDL, thrombosis, high blood pressure, smoking, and drinking (Table 1).

\subsection{Association of Individual Factors with Tortuosity}

As shown in Table 2, the univariable linear regression analysis for both the weighted mean and the standard deviation of tortuosity. Older age, longer duration, higher baPWV, and diagnosed with high blood pressure, thrombosis or Any DR were significantly associated with higher weighted mean and standard deviation tortuosity (all $P$ values $<0.05$ ). Moreover, we found that higher urine microalbuminuria and urine albumin/creatinine ratio significantly correlated with both higher tortuosity measures (all $P$ values $<0.05)$.

Table 3 and Table 4 showed the multivariable-adjusted linear regression analyses in three models. In model 1, patients with older age, or longer duration of diabetes had significantly higher tortuosity values both in the weighted mean and the standard deviation of tortuosity (all $P$ values $<0.05$ ), after adjustment for LDL, HDL, and diagnosed high blood pressure. In model 2 , we added factors related kidney failure and DR to the variables of model 1 . Higher tortuosity values were significantly associated with older age, longer duration, higher urine albumin/creatinine ratio (all $P$ values $<0.05$ ), while LDL was found negatively correlated with tortuosity. These associations remained even after excluding the factor of DR in model 3.

\section{Discussion}

In our current study, age, diabetes duration and urine albumin/creatinine ratio were independent risk factors of retinal vascular tortuosity, even after adjustment for HDL, LDL, high blood pressure, blood creatinine and DR.

Previous epidemiologic studies suggested an effect of age on retinal vascular tortuosity. Unlike Cheung et al. and Sasongko et al. [9] [10] finding a negative association between age and tortuosity, we demonstrated that age was positively associated with vascular tortuosity as an independent risk factor. This difference may come from the fact that Cheung's study was performed in a healthy population and Sasongko's study only included patients aged 12 - 20 years with type 1 diabetes. Moreover, these studies were conducted in different ethnicities compared to our patients. It has been proven earlier [20] that differences exist between ethnicities in vascular tortuosity values.

Longer duration was associated with higher tortuosity, even after adjustment for LDL, HDL, high blood pressure, blood creatinine and DR. This is in agreement with the study of Zoungas et al. who found that duration of diabetes is independently associated with micro-vascular complications [21]. This reveals that the duration of diabetes reflects the total glycemic control and risk factor exposure over time.

A high urine albumin/creatinine ratio was found to be associated with high vascular 
Table 1. Baseline characteristics and comparison among type 2 diabetic patients between Any DR and No DR groups.

\begin{tabular}{|c|c|c|c|c|}
\hline & \multicolumn{3}{|c|}{ Groups } & \multirow[b]{2}{*}{$P$ value } \\
\hline & Total $(n=504)$ & Any DR $(\mathrm{n}=386)$ & No DR $(n=118)$ & \\
\hline Gender & & & & 0.770 \\
\hline Male & $270(57.6)$ & $208(57.9)$ & $62(56.4)$ & \\
\hline Female & $199(42.4)$ & $151(42.1)$ & $48(43.6)$ & \\
\hline Age (years) & $51.4 \pm 12.8$ & $52.0 \pm 11.9$ & $49.2 \pm 15.4$ & 0.041 \\
\hline Duration (years) & $6.97 \pm 6.57$ & $7.36 \pm 6.82$ & $5.81 \pm 5.62$ & 0.045 \\
\hline Cholesterol (mmol/L) & $4.75 \pm 1.13$ & $4.80 \pm 1.14$ & $4.58 \pm 1.06$ & 0.080 \\
\hline Triglyceride & & & & 0.936 \\
\hline$\leq 1.69 \mathrm{mmol} / \mathrm{L}$ & $181(40.0)$ & 139(39.9) & $42(40.4)$ & \\
\hline$>1.69 \mathrm{mmol} / \mathrm{L}$ & $271(60.0)$ & $209(60.1)$ & $62(59.6)$ & \\
\hline $\mathrm{LDL}(\mathrm{mmol} / \mathrm{L})$ & $2.88 \pm 0.86$ & $2.90 \pm 0.87$ & $2.81 \pm 0.81$ & 0.352 \\
\hline No & $452(89.7)$ & $346(89.6)$ & $106(89.8)$ & \\
\hline $\mathrm{FBS}(\mathrm{mmol} / \mathrm{L})$ & $9.32 \pm 3.45$ & $9.36 \pm 3.58$ & $9.20 \pm 2.92$ & 0.679 \\
\hline Urea $(\mathrm{mmol} / \mathrm{L})$ & $5.40 \pm 1.79$ & $5.40 \pm 1.86$ & $5.43 \pm 1.56$ & 0.866 \\
\hline Urine microalbuminuria & & & & 0.231 \\
\hline$\leq 1.9 \mathrm{mg} / \mathrm{dL}$ & $287(65.7)$ & $217(64.2)$ & $70(70.7)$ & \\
\hline$>1.9 \mathrm{mg} / \mathrm{dL}$ & $150(34.3)$ & $121(35.8)$ & $29(29.3)$ & \\
\hline Urine albumin/creatinine ratio & & & & 0.074 \\
\hline$\leq 30$ & $318(72.2)$ & $239(70.7)$ & $79(79.8)$ & \\
\hline$>30$ & $119(27.8)$ & $99(29.3)$ & $20(20.2)$ & \\
\hline High blood pressure & & & & 0.970 \\
\hline Yes & $200(39.7)$ & $153(39.6)$ & $47(39.8)$ & \\
\hline No & $304(60.3)$ & $233(60.4)$ & $71(60.2)$ & \\
\hline SBP (mmHg) & $132.7 \pm 16.3$ & $134.9 \pm 16.5$ & $126.4 \pm 14.0$ & 0.002 \\
\hline DBP (mmHg) & $79.4 \pm 10.5$ & $80.9 \pm 10.6$ & $75.2 \pm 8.8$ & 0.001 \\
\hline Current Smoking & & & & 0.712 \\
\hline Yes & $178(35.3)$ & $138(35.8)$ & $40(33.9)$ & \\
\hline No & $326(64.7)$ & $248(64.2)$ & $78(66.1)$ & \\
\hline Current Drinking & & & & 0.216 \\
\hline Yes & $92(18.3)$ & $75(19.4)$ & $17(14.4)$ & \\
\hline No & $412(81.7)$ & $311(80.6)$ & $101(85.6)$ & \\
\hline \multicolumn{5}{|l|}{ Tortuosity $\left(\times 10^{3}\right)$} \\
\hline weighted mean & $12.05 \pm 1.66$ & $12.15 \pm 1.65$ & $11.72 \pm 1.67$ & 0.013 \\
\hline standard deviation & $24.31 \pm 3.69$ & $24.49 \pm 3.61$ & $23.69 \pm 3.92$ & 0.038 \\
\hline
\end{tabular}

Data are presented as mean \pm standard deviation for continuous variables and as number (percentage) for categorical variables. HbAlc, glycated hemoglobin; BMI, body mass index; LDL, low density lipoprotein; HDL, high density lipoprotein; CVD, Cerebral vascular disease; FBS, Fasting Blood Sugar; baPWV, brachial ankle pulse wave velocity; SBP, systolic blood pressure; DBP, diastolic blood pressure; DR, diabetic retinopathy. ${ }^{\star} \mathrm{P}<0.05$ significant. 
Table 2. Univariable regression analysis with the weighted mean and the standard deviation of tortuosity.

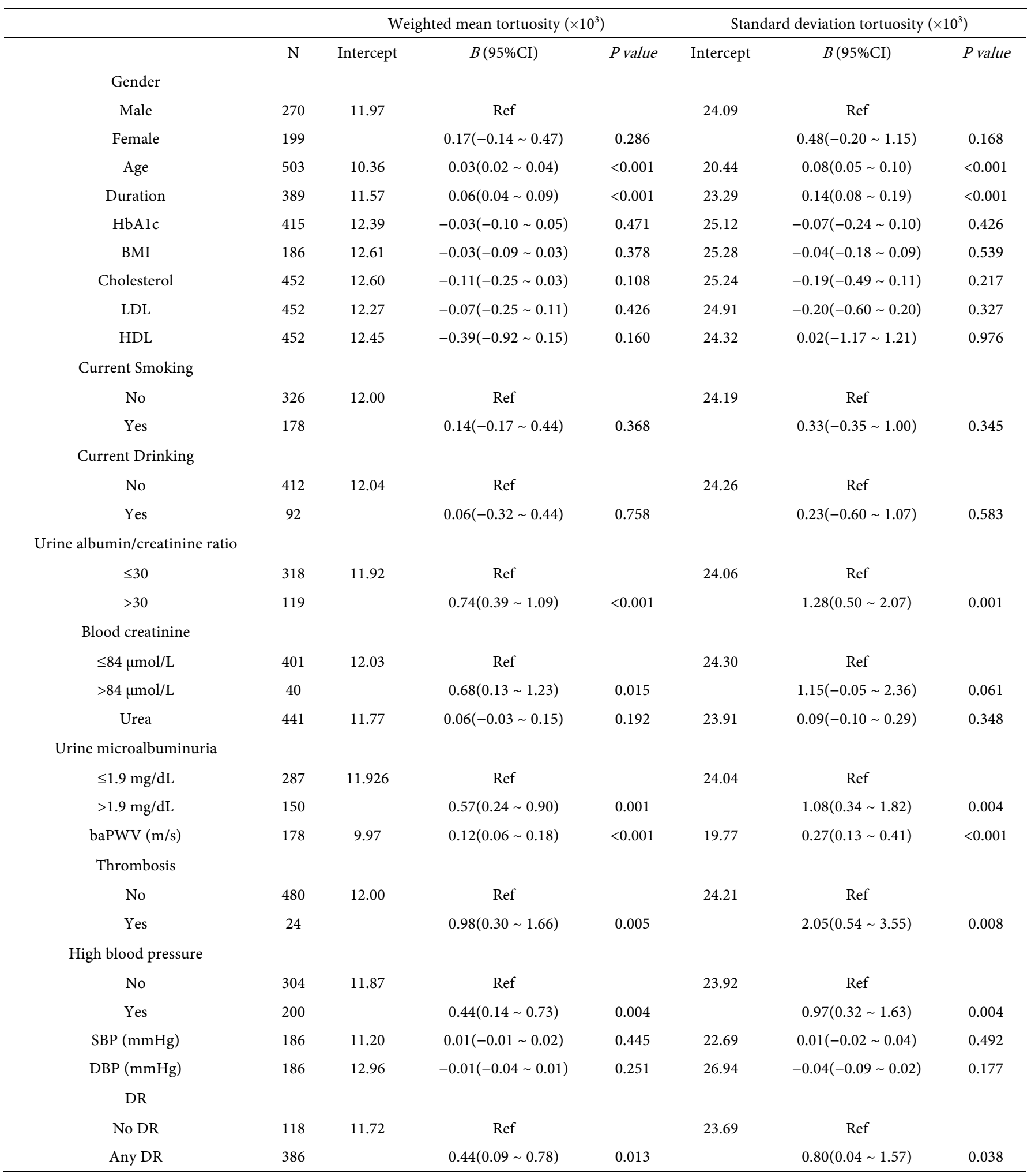

HbAlc, glycated hemoglobin; BMI, body mass index; LDL, low density lipoprotein; HDL, high density lipoprotein; baPWV, brachial ankle pulse wave velocity; SBP, systolic blood pressure; DBP, diastolic blood pressure; DR, diabetic retinopathy; N, number of subjects; B, regression coefficients; CI, confidence interval. ${ }^{\star P}<0.05$ significant. 
Table 3. Multivariable regression analysis with the weighted mean of tortuosity.

\begin{tabular}{|c|c|c|c|c|c|c|c|c|c|}
\hline \multicolumn{10}{|c|}{ Weighted mean tortuosity $\left(\times 10^{3}\right)$} \\
\hline & \multicolumn{3}{|c|}{ Model 1} & \multicolumn{3}{|c|}{ Model 2} & \multicolumn{3}{|c|}{ Model 3} \\
\hline & $B$ & $95 \% \mathrm{CI}$ & $P$ value & $B$ & $95 \% \mathrm{CI}$ & $P$ value & $B$ & $95 \% \mathrm{CI}$ & $P$ value \\
\hline Age & 0.03 & $0.01 \sim 0.04$ & 0.003 & 0.03 & $0.01 \sim 0.06$ & 0.007 & 0.23 & $0.01 \sim 0.43$ & 0.002 \\
\hline Duration & 0.04 & $0.01 \sim 0.07$ & 0.013 & 0.03 & $0.00 \sim 0.07$ & 0.037 & 0.03 & $0.00 \sim 0.06$ & 0.040 \\
\hline LDL & -0.21 & $-0.44 \sim 0.02$ & 0.070 & -0.24 & $-0.47 \sim-0.02$ & 0.037 & -0.25 & $-0.48 \sim-0.02$ & 0.031 \\
\hline \multicolumn{10}{|c|}{ High blood pressure } \\
\hline No & Ref & & & Ref & & & Ref & & \\
\hline Yes & 0.24 & $-0.16 \sim 0.64$ & 0.238 & 0.19 & $-0.21 \sim 0.58$ & 0.348 & 0.18 & $-0.21 \sim 0.57$ & 0.367 \\
\hline \multicolumn{10}{|c|}{ Urine albumin/creatinine ratio } \\
\hline$\leq 30$ & - & - & - & Ref & & & & & \\
\hline$>84 \mu \mathrm{mol} / \mathrm{L}$ & - & - & - & 0.47 & $-0.25 \sim 1.19$ & 0.200 & 0.48 & $-0.23 \sim 1.20$ & 0.186 \\
\hline \multicolumn{10}{|l|}{$\mathrm{DR}$} \\
\hline No DR & - & - & - & Ref & & & - & - & - \\
\hline Any DR & - & - & - & 0.23 & $-0.38 \sim 0.84$ & 0.465 & - & - & - \\
\hline
\end{tabular}

LDL, low density lipoprotein; HDL, high density lipoprotein; DR, diabetic retinopathy; N, number of subjects; B, regression coefficients; CI, confidence interval. Model 1: Model with age, duration, LDL, HDL, diagnosed high blood pressure $(\mathrm{N}=298)$. Model 2: Model with age, duration, LDL, HDL, diagnosed high blood pressure, urine albumin creatinine ratio, blood creatinine and DR $(\mathrm{N}=298)$. Model 3: Model with age, duration, LDL, HDL, diagnosed high blood pressure, urine albumin creatinine ratio, and blood creatinine $(\mathrm{N}=298)$.

Table 4. Multivariable regression analysis with the standard deviation of tortuosity.

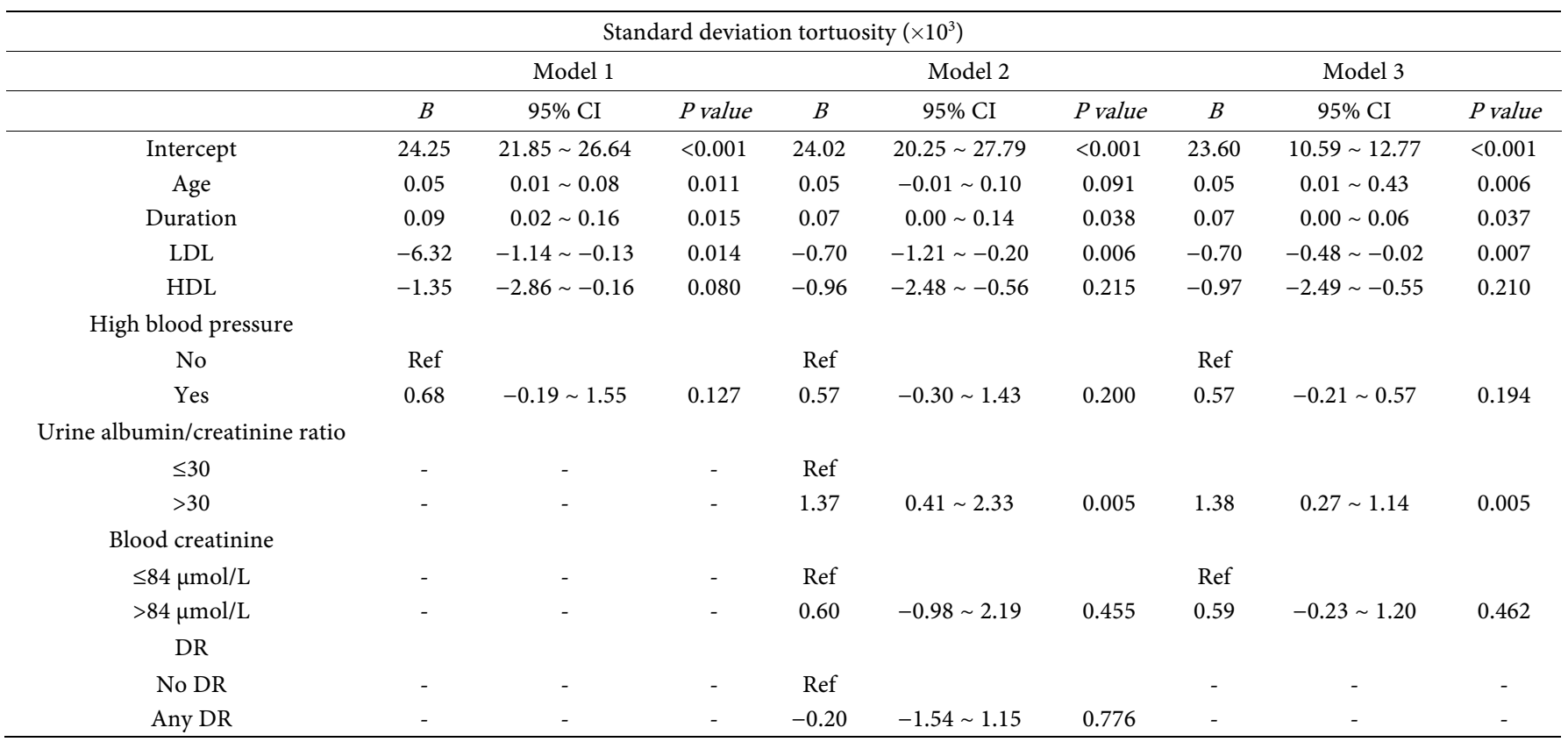

LDL, low density lipoprotein; HDL, high density lipoprotein; DR, diabetic retinopathy; N, number of subjects; B, regression coefficients; CI, confidence interval. Model 1: Model with age, duration, LDL, HDL, diagnosed high blood pressure $(\mathrm{N}=298)$. Model 2: Model with age, duration, LDL, HDL, diagnosed high blood pressure, urine albumin creatinine ratio, blood creatinine and DR $(\mathrm{N}=298)$. Model 3: Model with age, duration, LDL, HDL, diagnosed high blood pressure, urine albumin creatinine ratio, and blood creatinine $(\mathrm{N}=298)$. 
tortuosity independently. Associations between diabetic neuropathy and retinal microvascular changes have been demonstrated in previous studies [22] [23]. Although the underlying mechanisms for these vascular changes were not completely understood, our finding is new, and indicates that increased retinal vascular tortuosity is one of the early microvascular alterations in type 2 diabetic patients, or at the early stage of the diabetic microvascular complications.

The tortuosity measures were found significantly higher in patients with DR, as in univariable linear regression analyses DR was significantly associated with both tortuosity measures. An experimental study in a galactose-fed rat model demonstrated a positive association between retinal vascular tortuosity and diabetic-like retinopathy [13]. Sasongko et al. also found that increased arteriolar tortuosity was associated with mild and moderate stages of DR [5]. Kristinsson et al. showed that patients with DR and diabetic macular edema had more tortuous retinal vessels [7]. Although the tortuosity measurement method in our study was different from the study by Kristinsson, similar associations were observed, which suggests that diabetic patients with DR are more likely to have tortuous retinal vessels. But in multivariable linear regression analyses, the association between tortuosity and DR disappeared, after adjustment for age, duration, HDL, LDL, high blood pressure, urine albumin/creatinine ratio and blood creatinine. In our current study, we could not calculate a tortuosity value for arteries and veins separately. If these measures would have been available, there could have been an association between tortuosity and DR independently.

Strengths of our study are: a novel robust and fully automatic quantitative method was used to measure retinal vascular tortuosity with high reliability, which has better accuracy than qualitative assessment by trained ophthalmologists; the retinal fundus photographs are taken with nonmydriatic, fovea-centered, by a laser-scanning ophthalmoscope (SLO) camera, which can avoid patients' uncomfortable feeling after dilation, and generates a high contrast between retinal vessels and their context.

However, some limitations should also be noted. First, the patients in our study are hospitalized in one hospital, so they can only be regarded as a representative sample of severe type 2 diabetes. We should be careful with extrapolating the results to the whole type 2 diabetes population. Second, this cross-sectional study cannot provide temporal information on the association, so longitudinal studies are needed. In addition, DR assessment in our study is only divided into No DR and Any DR, which may eliminate some associations between tortuosity and a detailed grading of DR. Lastly, the tortuosity measures are performed in retinal arteries and veins together, which makes that we cannot evaluate the associations between tortuosity and factors in arteries and veins respectively. Given their different vessel wall physiology, these associations may be more significant and predictive.

\section{Conclusion}

In conclusion, we showed that quantitative measurement of retinal vascular tortuosity was independently associated with older age, longer duration, and higher urine albu- 
$\mathrm{min} /$ creatinine ratio in Chinese hospitalized type 2 diabetic patients, regardless of the presence of DR. These findings may offer new insights into early retinal microvascular changes and diabetic microvascular complications, like diabetic retinopathy and diabetic neuropathy. This fully automated cost-effective quantitative measurement method offers great potential in screening diabetic patients for microvascular complications.

\section{References}

[1] Hu, F.B. (2011) Globalization of Diabetes: the Role of Diet, Lifestyle, and Genes. Diabetes Care, 34, 1249-1257. http://dx.doi.org/10.2337/dc11-0442

[2] Xu, Y., Wang, L., He, J., Bi, Y., Li, M., Wang, T., et al. (2013) Prevalence and Control of Diabetes in Chinese Adults. Journal of American Medical Association, 310, 948-959. http://dx.doi.org/10.1001/jama.2013.168118

[3] Aguriee, F., Brown, A., Cho, N.H., Dahlquist, G., Dodd, S., Dunning, T., et al. (2013) International Diabetes Federation (IDF) Diabetes Atlas. 6th Edition, International Diabetes Federation.

[4] Cheung, C.Y., Ikram, M.K., Sabanayagam, C. and Wong, T.Y. (2012) Retinal Microvasculature as a Model to Study the Manifestations of Hypertension. Hypertension, 60, 10941103. http://dx.doi.org/10.1161/HYPERTENSIONAHA.111.189142

[5] Sasongko, M.B., Wong, T.Y., Nguyen, T.T., Cheung, C.Y., Shaw, J.E. and Wang, J.J. (2011) Retinal Vascular Tortuosity in Persons with Diabetes and Diabetic Retinopathy. Diabetologia, 54, 2409-2416. http://dx.doi.org/10.1007/s00125-011-2200-y

[6] Kiely, A.E., Wallace, D.K., Freedman, S.F. and Zhao, Z. (2010) Computer Assisted Measurement of Retinal Vascular Width and Tortuosity in Retinopathy of Prematurity. Archives of Ophthalmology, 128, 847-852. http://dx.doi.org/10.1001/archophthalmol.2010.133

[7] Kristinsson, J.K., Gottfredsdottir, M.S. and Stefansson, E. (1997) Retinal Vessel Dilatation and Elongation Precedes Diabetic Macular Oedema. British Journal of Ophthalmology, 81, 274-278. http://dx.doi.org/10.1136/bjo.81.4.274

[8] Cheung, C.Y., Lamoureux, E., Ikram, M.K., Sasongko, M.B., Ding, J., Zheng, Y., et al. (2012) Retinal Vascular Geometry in Asian Persons with Diabetes and Retinopathy. Journal of Diabetes Science and Technology, 6, 595-605. http://dx.doi.org/10.1177/193229681200600315

[9] Cheung, C.Y., Zheng, Y., Hsu, W., Lee, M.L., Lau, Q.P., Mitchell, P., et al. (2011) Retinal Vascular Tortuosity, Blood Pressure, and Cardiovascular Risk Factors. Ophthalmology, 118, 812-818. http://dx.doi.org/10.1016/j.ophtha.2010.08.045

[10] Sasongko, M.B., Wang, J.J., Donaghue, K.C., Cheung, N., Benitez-Aguirre, P., Jenkins, A.J., et al. (2010) Alterations in Retinal Microvascular Geometry in Young Type 1 Diabetes. Diabetes Care, 33, 1331-1336. http://dx.doi.org/10.2337/dc10-0055

[11] Sasongko, M.B., Wong, T.Y., Donaghue, K.C., Cheung, N., Jenkins, A.J., Benitez-Aguirre, P., et al. (2011) Retinal Arteriolar Tortuosity Is Associated with Retinopathy and Early Kidney Dysfunction in Type 1 Diabetes. American Journal of Ophthalmology, 153, 176183. http://dx.doi.org/10.1016/j.ajo.2011.06.005

[12] Benitez-Aguirre, P., Craig, M.E., Sasongko, M.B., Jenkins, A.J., Wong, T.Y., Wang, J.J., et al. (2011) Retinal Vascular Geometry Predicts Incident Retinopathy in Young People with Type 1 Diabetes: A Prospective Cohort Study from Adolescence. Diabetes Care, 34, 16221627. http://dx.doi.org/10.2337/dc10-2419 
[13] Robison Jr, W.G., Laver, N.M., Jacot, J.L. and Glover, J.P. (1995) Sorbinil Prevention of Diabetic-Like Retinopathy in the Galactose-Fed Rat Model. Investigative Ophthalmology \& Visual Science, 36, 2368-2380.

[14] Bekkers, E.J., Zhang, J., Duits R. and ter Haar Romeny, B.M. (2015) Curvature Based Biomarkers for Diabetic Retinopathy via Exponential Curve Fits in SE(2). In: Chen, X., Garvin M.K., Liu, J., Trucco, E. and Xu, Y., Eds., Proceedings of the Ophthalmic Medical Image Analysis Second International Workshop, OMIA 2015, Munich, 9 October 2015, 113-120.

[15] Duits, R. and Franken, E. (2010) Left-Invariant Parabolic Evolutions on SE (2) and Contour Enhancement via Invertible Orientation Scores. Part II: Non-Linear Left-Invariant Diffusions on Invertible Orientation Score. Quarterly of Applied Mathematics, 68, 293-331. http://dx.doi.org/10.1090/S0033-569X-10-01173-3

[16] Duits, R., Janssen, M., Hannink, J. and Sanguinetti, G. (2015) Locally Adaptive Frames in the Roto-Translation Group and their Applications in Medical Imaging. Journal of Mathematical Imaging \&Vision, 1-36.

[17] Franken, E. and Duits, R. (2009) Crossing-Preserving Coherence-Enhancing Diffusion on Invertible Orientation Scores. International Journal of Computer Vision, 85, 253-278. http://dx.doi.org/10.1007/s11263-009-0213-5

[18] Bekkers, E., Duits, R., Berendschot, T., and ter Haar Romeny, B. (2014) A Multi-Orientation Analysis Approach to Retinal Vessel Tracking. Journal of Mathematical Imaging \& Vision, 49, 583-610. http://dx.doi.org/10.1007/s10851-013-0488-6

[19] Wong, T.Y., Cheung, N., Tay, W.T., Wang, J.J., Aung, T. and Saw, S.M. (2008) Prevalence and Risk Factors for Diabetic Retinopathy: The Singapore Malay Eye Study. Ophthalmolo$g y, 115,1869-1875$. http://dx.doi.org/10.1016/j.ophtha.2008.05.014

[20] Li, X., Wong, W.L., Cheung, C.Y., Cheng, C.Y., Ikram, M.K., Li, J., et al. (2013) Racial Differences in Retinal Vessel Geometric Characteristics: A Multiethnic Study in Healthy Asians. Investigative Ophthalmology \& Visual Science, 54, 3650-3656. http://dx.doi.org/10.1167/iovs.12-11126

[21] Zoungas, S., Woodward, M., Li, Q., Cooper, M.E., Hamet, P., Harrap, S., et al. (2014) Impact of Age, Age at Diagnosis and Duration of Diabetes on the Risk of Macrovascular and Microvascular Complications and Death in Type 2 Diabetes. Diabetologia, 57, 2465-2474. http://dx.doi.org/10.1007/s00125-014-3369-7

[22] Wong, T.Y., Coresh, J., Klein, R., Muntner, P., Couper, D.J., Sharrett, A.R., et al. (2004) Retinal Microvascular Abnormalities and Renal Dysfunction: the Atherosclerosis Risk in Communities Study. Journal of the American Society of Nephrology, 15, 2469-2476. http://dx.doi.org/10.1097/01.ASN.0000136133.28194.E4

[23] Edwards, M.S., Wilson, D.B., Craven, T.E., Stafford, J., Fried, L.F., Wong, T.Y., et al. (2005) Associations between Retinal Microvascular Abnormalities and Declining Renal Function in the Elderly Population: The Cardiovascular Health Study. American Journal of Kidney Diseases, 46, 214-224. http://dx.doi.org/10.1053/j.ajkd.2005.05.005 
Submit or recommend next manuscript to SCIRP and we will provide best service for you:

Accepting pre-submission inquiries through Email, Facebook, LinkedIn, Twitter, etc.

A wide selection of journals (inclusive of 9 subjects, more than 200 journals)

Providing 24-hour high-quality service

User-friendly online submission system

Fair and swift peer-review system

Efficient typesetting and proofreading procedure

Display of the result of downloads and visits, as well as the number of cited articles

Maximum dissemination of your research work

Submit your manuscript at: http://papersubmission.scirp.org/

Or contact jbise@scirp.org 